\title{
A NOVEL SIGMA 2 RECEPTOR AGONIST INDUCES APOPTOSIS IN HUMAN SK-MEL-2 CELL LINE
}

\author{
L. Benassi ${ }^{1,2}$, C. Vaschieri ${ }^{1,2}$, P. Azzoni ${ }^{1,2}$, C. Magnoni1 ${ }^{1,2}$, A. Pisciotta ${ }^{1}$, L.Bertoni ${ }^{1}$, G. Carnevale ${ }^{1}$, S.Franchini ${ }^{3}$, C. Sorbi $^{3}$, \\ G.Pellacani ${ }^{1,2}$.
}

\section{Department of Surgery, Medicine, Dentistry and Morphological Sciences with interest in Transplant, Oncology and Regenerative Medicine, University of Modena and Reggio Emilia. Italy. \\ 2.Laboratory of Dermatology, University of Modena and Reggio Emilia. Italy. 3. Department of Life Sciences, University of Modena and Reggio Emilia. Italy.}

Introduction

Melanoma is a highly metastatic tumor and the most deadly dermatologic malignancy because the disease is frequently diagnosed in the advanced stage. Metastasized melanoma is refractory to current therapeutic modalities. Melanomas possess moderate to high levels of $\sigma$ receptors as shown by a pronounced uptake of $\sigma$ several ligands labeled for PET or SPECT.

The sigma receptors are a pharmacologically defined family of membrane bound receptors that bind compounds from a variety of structural classes. Two subtypes of sigma receptor have been identified: the sigma-1 receptor $(\sigma 1)$ is a $25-\mathrm{kDa}$ protein that is known to promote cell survival. In contrast, the sigma-2 receptor $(\sigma 2)$ has not been cloned so far and it is a $21.5-\mathrm{kDa}$ protein. The $\sigma 2$ receptors were found to be more expressed than that of the $\sigma 1$ receptors. The density of the $\sigma 2$ receptors are about 10-fold higher in rapidly proliferating cancer cells than that in quiescent cancers cells, compared with noncancerous tissue. The $\sigma 2$ receptors has been validated in an extensive list of human and rodent cancer cell lines and tumors, and thus a ubiquitous role in cancer biology has been proposed

Moreover, $\sigma 2$ receptor ligands can rapidly internalize into tumor cells and activate cell death via multiple pathways indicating that multiple mechanism exists aside from $\sigma 2$ receptor ligands-induced cell death. Thus, the $\sigma 2$ receptor may both serve as a receptor-based biomarker to distinguish different proliferative states of solid tumors and as a promising target for the treatment of cancer. Previous works have shown that the exposure to $\sigma 2$ receptor agonists results in morphological changes and apoptosis in various cell tumor lines.

Aim

Our study aimed to identify, among several classes of $\sigma 1$ and $\sigma 2$ ligands developed in our laboratories, the optimal compound for evaluating the inhibition of cell proliferation and cell death/apoptosis induction in two p53-mutant melanoma cell lines, i.e. SK-MEL-2 and SK-MEL-28.

Results

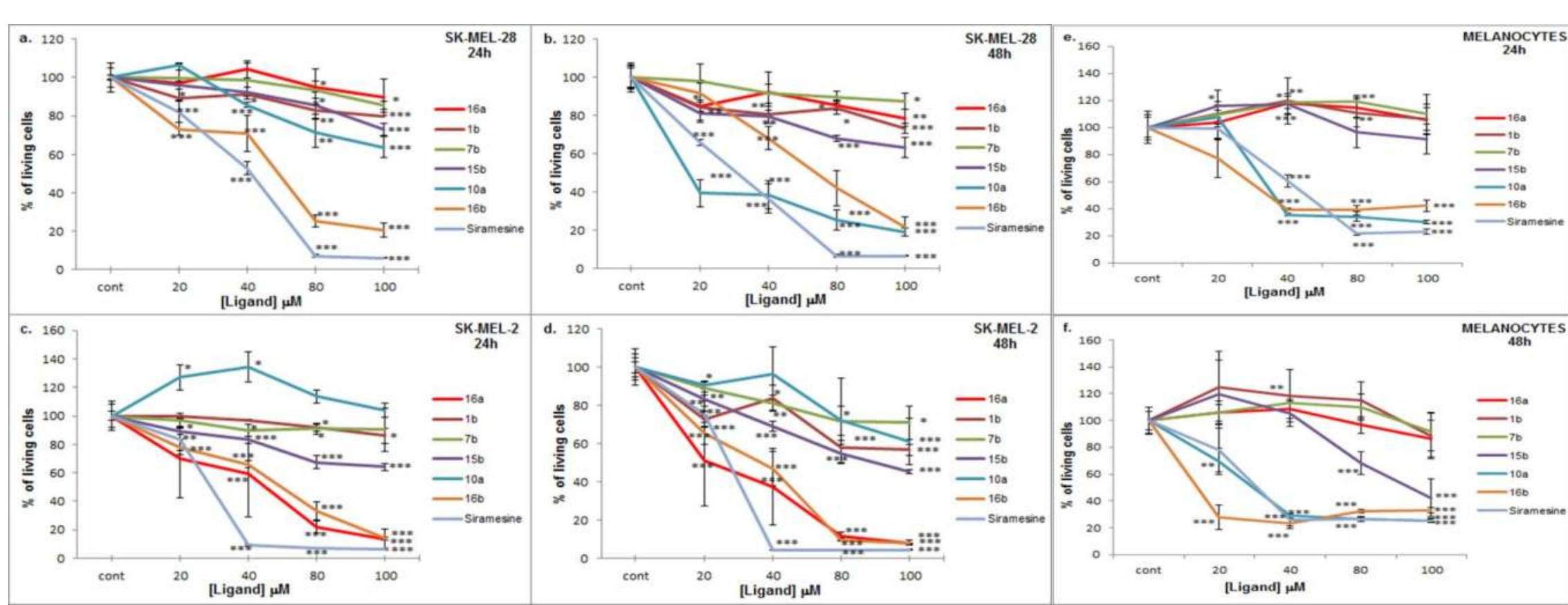

Fig.1 MTT assay. Dose-response curves obtained in SK-MEL-28 (a, b), SK-MEL-2 (c, d) and healthy human melanocytes (e, f) after 24 and 48 hours of treatment. The 16a compound induced inhibition in cell growth in SK-MEL-2 but did not affect cell viability in SK-MEL-28 and healthy human melanocytes. Siramesine was used as positive control.
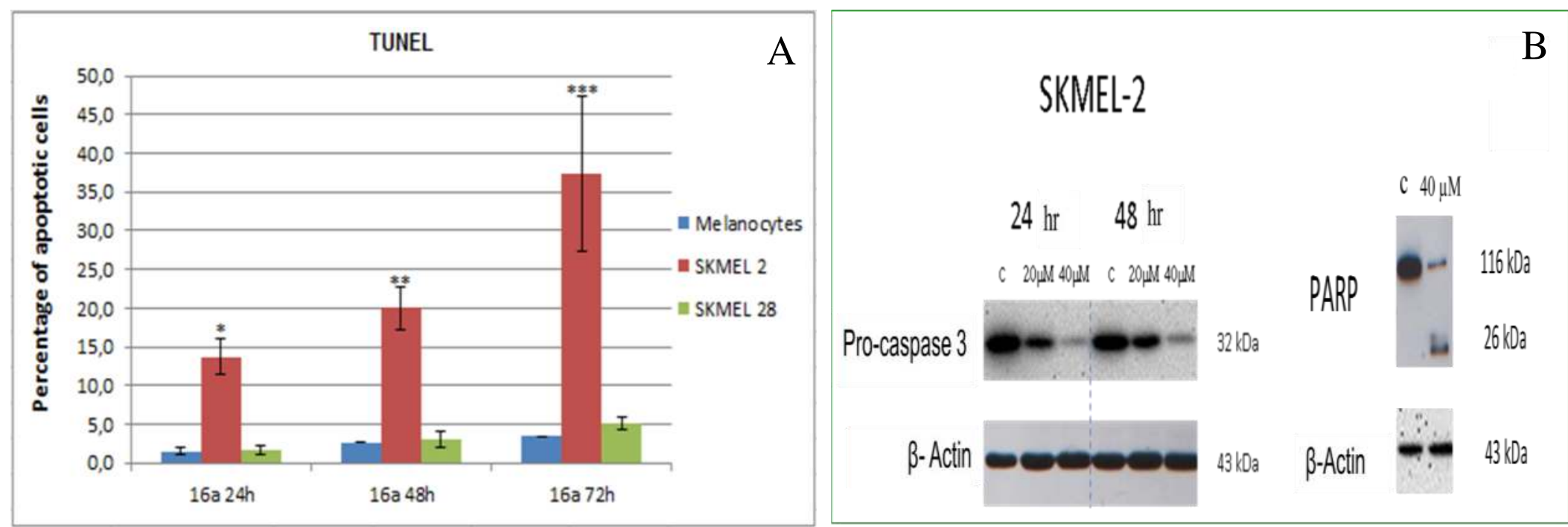

Fig.3 (A) TUNEL test after treatment with16a. SK-MEL-2 cells stained positive for TUNEL test, reaching a peak value $(\sim 37 \%)$ at 72 hours. (B) Western blot analysis of proCaspase 3 and PARP in SK-MEL-2 treated with16a. pro-Caspase 3 expression and PARP cleavage were detected in SK-MEL-2 following exposure to 16a.

\section{Conclusion}

The compound 16a exhibited $\sigma_{2} \mathrm{R}$ selectivity and was proven to behave as a $\sigma_{2} \mathrm{R}$ agonist, being able to induce an anti-proliferative activity towards SK-MEL-2, a metastatic melanoma cell line, whilst not affecting healthy human melanocytes. These results are consistent with sigma-2 receptor-mediated apoptosis via p53-independent mechanism. The cleavage of downstream caspases and PARP are not the only consequence of the $\sigma_{2}$ receptor pro-apoptotic mechanism triggered in SK-MEL-2 cells. Indeed, we observed that the anti-cancer effect was also supported by the induction of oxidative stress and massive lysosomal permeabilization. Further studies are needed to investigate further mechanisms involved with cell death, such as autophagy and cell-cycle regulation. 16a might be a promising candidate for development of novel metastatic melanoma therapies. The induction of apoptosis upon activation combined with endogenous cancer cell selectivity makes the $\sigma_{2}$ receptor an attractive target for chemotherapeutic intervention.

References

X. Han, Y. Han, Y. Zheng, Q. Sun, T. Ma, et al. PLOS ONE (2017) 18(4).

C. Zeng, J. Rothfuss, J. Zhang, W. Chu, S. Vangveravong, Z. Tu, F. Pan, K.C. Chang, R. Hotchkiss and R.H. Mach. Br J Cancer (2012)14;106(4):693-701

C. Zeng, J.M. Rothfuss, J. Zhang, S. Vangveravong, W. Chu, S. Li, Z.Tu, J. Xu, R.H. Mach. Analytical Biochemistry (2014) 448 68-74. 